\title{
A Relação entre Sintomas Depressivos e Habilidades Sociais em Adolescentes
}

\author{
Maria Luzia Silva Santana ${ }^{1}$; Cláudia Cristina Fukuda ${ }^{2}$; Erenice Natalia Soares de Carvalho ${ }^{3}$
}

\begin{abstract}
Resumo: Essa pesquisa descreve a relação entre sintomas depressivos e o repertório de habilidades sociais em adolescentes, relacionando os níveis de depressão à frequência e dificuldades de habilidades sociais. Participaram 153 adolescentes com idades entre 12 e 17 anos de uma escola pública localizada em Planaltina, no Distrito Federal. Quatro participantes apresentaram nível moderado de sintomas depressivos e cinco adolescentes, nível grave. Desses, sete foram do sexo feminino. Encontrou-se diferenças entre os níveis de sintomas depressivos entre os sexos, sendo que as adolescentes apresentaram índices de sintomatologia depressiva mais elevada que os adolescentes. Não houve diferenças significativas entre as médias nos escores de frequência e de dificuldade de habilidades sociais entre os sexos. Encontrou-se uma correlação inversa entre depressão e frequência de habilidades sociais e uma correlação positiva entre depressão e dificuldade de habilidades sociais, o que sugere uma relação entre sintomas depressivos e repertório de habilidades sociais em adolescentes.
\end{abstract}

Palavras-chave: Sintomas Depressivos. Habilidades Sociais. Adolescentes.

\section{The Relationship Between Depressive Symptoms and Social Skills in Adolescents}

\begin{abstract}
This research describes the relationship between the depressive symptoms and the social skills repertoire in adolescents, relating depression levels to the frequency and social skills difficulties. We studied 153 teenagers between the ages of 12 and 17 in a public school located in Planaltina, in the Distrito Federal. Four participants had a moderate level of depressive symptoms and five adolescents, a serious level. Of these, seven were female. We have found differences between the levels of depressive symptoms and the gender, and the girls presented higher rates of depressive symptoms than the boys. There were no significant differences between the frequency and the social skills difficulty scores between the genders. There was an inverse correlation between depression and social skills frequency and a positive correlation between depression and social skills difficulty, suggesting a relationship between depressive symptoms and social skills repertoire in adolescents.
\end{abstract}

Keywords: Depressive Symptoms. Social skills. Teenagers.

\footnotetext{
${ }^{1}$ Doutoranda e Mestra em Psicologia (Universidade Católica de Brasília- UCB); Especialista em Educação e Promoção da Saúde (Universidade de Brasília - UNB) e em Psicologia Social (Universidade Estadual de Santa Cruz-UESC); Psicóloga (Centro de Ciências da Saúde da Universidade Federal do Recôncavo da Bahia- UFRB); Licenciatura Plena em História (Universidade do Estado da Bahia -UNEB) e em Pedagogia (Faculdades Alfredo Nasser - UNIFAN). É Professora Assistente (DE) na Universidade Federal de Mato Grosso do Sul UFMS. Contato: santanapsi@gmail.com;

${ }^{2}$ Doutora em Psicologia pela Universidade de Brasília - UnB- Brasil. Pós-Doutorando em Universidade do Porto, UP - Portugal. Docente no Programa de Mestrado e Doutorado em Psicologia da Universidade Católica de Brasília - UCB/DF - Brasil. Contato: fukuda@ucb.br; ${ }^{3}$ Doutora e Mestra em Psicologia pela Universidade de Brasília - UnB- Brasil. Federação Nacional das APAES/UNIAPE, FENAPAES, Brasil. Contato: erenicedasilvaoliveira@gmail.com.
} 


\section{Introdução}

Estudos sugerem que a depressão está associada ao déficit de habilidades sociais em adolescentes (CAMPOS, 2009; GRESHAM; COOK; CREWS, 2004; HOROWITZ, 2007; ROCKHILL et al., 2009; YOUNG; MUFSON; GALLOP, 2010). Nesse sentido, Tavares (2005) salienta que pessoas com poucas habilidades sociais podem desenvolver problemas psicológicos, dentre eles, timidez excessiva, síndrome do pânico, dificuldades sexuais e depressão. Aquelas com depressão tendem a demonstrar déficits de habilidades sociais e relações interpessoais pobres (FERNANDES; FALCONE, 2009; SELIGMAN, 1977).

Considera-se que programas psicoeducativos com vista às interações sociais dos adolescentes poderão contribuir com a remissão de sintomas depressivos nessa população. Inclusive, sugere-se que o treino de habilidades sociais poderá possibilitar efeitos positivos nos adolescentes e familiares envolvidos no programa (SILVA; MURTA, 2009). Adolescentes deprimidos se beneficiam do treino de habilidades sociais, eles podem ser instruídos a observar seus pares e a imitar situações nas quais eles se sentem desconfortáveis ou inseguros devido às normas sociais esperadas (FRIDIBERG; McCLURE, 2004).

Del Prette e Del Prette (2010) salientam que as habilidades sociais integram um campo teórico complexo, cobrindo uma gama de temas como assertividade, não-assertividade, desempenho social e competência social. Os vocábulos desempenho social, habilidades sociais e competência social são centrais no campo do treino das habilidades sociais (DEL PRETTE; DEL PRETTE, 2008).

As habilidades sociais representam o universo mais abrangente das relações interpessoais, envolvendo um conjunto de comportamentos que estão presentes no repertório da pessoa e que facilitam seu relacionamento interpessoal. As habilidades sociais se estendem para além da assertividade, incluem as habilidades de comunicação, de resolução de problemas, de cooperação e aqueles próprios dos rituais sociais estabelecidos pelo contexto cultural (DEL PRETTE; DEL PRETTE, 1996, 2010).

Há um relativo consenso sobre os componentes das habilidades sociais (DEL PRETTE; DEL PRETTE, 2010). Do ponto de vista de Caballo (2010), esses componentes são organizados em comportamentais, fisiológicos e cognitivos. O componente cognitivo inclui as 
competências cognitivas, as estratégias de codificação e construtos pessoais, expectativas, valores subjetivos dos estímulos e sistemas e planos de autorregulação.

Conforme Caballo (2010) a percepção e a avaliação cognitiva da pessoa sobre as diversas situações, estímulos e acontecimentos estão determinadas por um sistema persistente integrado por abstrações e concepções do mundo, incluindo os conceitos de si mesmo de forma que "[...] a aprendizagem social do comportamento é mediada por processos cognitivos que, quando disfuncionais, influenciam negativamente no desempenho interpessoal " (DEL PRETTE; DEL PRETTE, 2000, p. 225).

Há evidências empíricas e clínicas de que a falta de repertório apropriado de habilidades sociais está relacionada à depressão. Além da etiologia multivariada da depressão, é reconhecido que os estados depressivos caracterizam-se, também, pela deteriorização das relações sociais (DEL PRETTE; DEL PRETTE, 2010).

Assim, adolescentes com indicadores de depressão apresentam comprometimento no repertório de habilidades sociais. De acordo com Segrin (2000), pode-se identificar três pressupostos na relação entre repertório deficitário de habilidades sociais e depressão: (a) repertório deficitário de habilidades sociais como causa de depressão; (b) depressão como causa do deficitário repertório de habilidades sociais; e (c) poucas habilidades sociais como fator de vulnerabilidade para o desenvolvimento de depressão. $\mathrm{O}$ pesquisador ainda pontua que, na atualidade, há evidências para sustentar cada uma dessas concepções e que a relação entre elas pode ter uma variedade de formas.

Campos (2010) caracterizou o repertório de habilidades sociais de 103 adolescentes com indicadores de depressão, sendo 75 meninas e 28 meninos com idades entre 12 a 14 anos. Nessa pesquisa, $52,4 \%$ dos participantes apresentaram repertório de habilidades sociais abaixo da média inferior e 10,7\% demonstraram repertório médio inferior de habilidades sociais. As adolescentes apresentaram repertório mais deficitário de habilidades sociais em todas as categorias investigadas: empatia, autocontrole, civilidade, assertividade, desenvoltura social, abordagem afetiva, enquanto os adolescentes apresentaram mais recursos comportamentais, como autocontrole, empatia, assertividade e abordagem afetiva.

Witvliet et al. (2010) realizou uma pesquisa com 310 crianças com a duração de três anos, com acompanhamento dos 11 aos 14 anos de idade e examinou se o isolamento de 
colegas aumentava os sintomas depressivos. Esses achados sugerem que o isolamento social é um dos fatores de risco para o desenvolvimento da depressão na adolescência.

Estudos que associam dificuldades interpessoais de adolescentes deprimidos a déficits de habilidades sociais foram realizados por Gresham, Cook e Crews (2004). Como descritos pelos autores, os adolescentes deprimidos, com frequência, expressaram sentimentos em momentos inapropriados e emitiram comportamentos indesejados. Consequentemente, distanciavam as pessoas de seu convívio, gerando rejeição social.

Em uma perspectiva psicopatológica, adolescentes com diagnóstico de depressão bipolar ficaram atrás de seus pares no desempenho de habilidades sociais. Em se tratando de dificuldade na regulação emocional, estudos demonstraram sua interferência no desenvolvimento de comportamentos sociais apropriados (GOLDSTEIN; MIKLOWITZ; MULLEN, 2006).

Nesse sentido, a pesquisa de Rockhill el al. (2009) examinou a relação entre competência social, apoio social e psicopatologia, incluindo a depressão e o transtorno de conduta, envolvendo uma amostra de 521 adolescentes, que foram agrupados nos grupos de risco: depressão, problemas de conduta e depressão com problemas de conduta. Os resultados sugeriram que os baixos níveis de competência social têm relações significativas no grupo de adolescentes com sintomas depressivos.

No que diz respeito aos aspectos interventivos, os pesquisadores sugeriram que a intervenção com vistas à melhoria da competência social e do apoio social poderia melhorar os resultados funcionais, especialmente para jovens com sintomas depressivos ou depressão e comorbidade. Por sua vez, a presença de determinadas classes de habilidades sociais pode contribuir para maximizar a competência social e funcionar como fator de proteção (CAMPOS, 2009).

Decerto a pesquisa de Young, Mufson e Gallop (2010) avaliou a eficácia de um programa de prevenção indicada para adolescentes deprimidos, incluindo 57 adolescentes com sintomas elevados de depressão. Os participantes da investigação foram randomizados em dois grupos: um grupo submeteu-se a psicoterapia interpessoal com o treinamento de habilidades sociais e o outro foi atendido em escola de aconselhamento. A partir dos resultados, notou-se que os adolescentes do grupo de psicoterapia interpessoal relataram taxas significativamente maiores de mudança nos sintomas de depressão e funcionamento em geral 
de que os adolescentes em aconselhamentona avaliação pós-intervenção. Os primeiros mostraram significativamente menos sintomas de depressão e melhor funcionamento geral.

Outro estudo, na temática anterior, foi realizado por Gunlicks-Stoessel et al. (2010), examinando aspectos do funcionamento de adolescentes deprimidos com o uso da psicoterapia interpessoal e o tratamento usual em clínicas baseadas na escola de saúde. Participaram do estudo 63 adolescentes com idade entre 12 a 18 anos, aleatoriamente designados para receber um dos tipos de acompanhamento. Os resultados indicaram que a aplicação da psicoterapia interpessoal beneficiou, particularmente, os adolescentes que relataram altos níveis de conflito com suas mães e disfunção social com amigos. A propósito, as intervenções que ensinam estratégias aos jovens para lidar com os eventos negativos da vida e estressores interpessoais podem ser especialmente úteis no tratamento e prevenção da depressão (HOROWITZ, 2007).

A revisão de literatura realizada neste trabalho aponta que o treinamento de habilidades sociais contribui com a remissão de sintomas depressivos em adolescentes. Aliás, demonstrou evidências da relação entre déficit nas habilidades sociais e depressão nessa população específica. Por outro lado, possibilitou a construção da hipótese que orienta o presente estudo, que reafirma a relação entre depressão e habilidades sociais e cuja contribuição consiste em investigar a hipótese da existência de uma relação entre os dois fenômenos, aliás, essa pesquisa buscou resposta para essa hipótese. Com base na hipótese, a presente pesquisa teve como objetivo descrever a relação entre sintomas depressivos e o repertório de habilidades sociais em adolescentes, relacionando os níveis de depressão à frequência e dificuldades de habilidades sociais.

\section{Material e Método}

A pesquisa foi iniciada após aprovação pelo Comitê de Ética em Pesquisa da Universidade Católica de Brasília- CEP/UCB, número 393.089. Durante o estudo, foi assegurado o sigilo dos participantes, o direito de desistir da pesquisa em qualquer momento sem nenhum prejuízo. O retorno do resultado aos participantes foi realizado mediante a entrega do relatório contendo informações referentes ao resultado da pesquisa. A coleta dos 
dados foi realizada com uma amostra de conveniência em uma escola pública localizada em Planaltina, no Distrito Federal.

\section{Participantes}

Os participantes foram 153 estudantes com idades entre 12 e 17 anos $(\bar{x}=13,6 ; d p=$ $1,1)$, desses $66,7 \%$ do sexo feminino e $33,3 \%$ do sexo masculino. Do total geral $126(82,4 \%)$ participantes tinham idades entre 13 a 15 anos. Do total geral dos participantes 41 (26,8\%) cursavam o $6^{\circ}$ ano e $112(73,2 \%)$, o $8^{\circ}$ ano. E $71(46,4 \%)$ frequentavam a escola no turno matutino e $82(53,6 \%)$ no turno vespertino.

\section{Instrumentos}

Questionário Sociodemográfico: contém 7 questões e foi utilizado para caracterizar os participantes da pesquisa nos aspectos sociais e escolares. A aplicação possibilitou obter informações sobre idade, gênero, família e aspectos econômicos.

Inventário de Depressão de Beck (BDI-II): tem como objetivo detectar sintomas depressivos conforme os critérios sintomáticos de Episódio Depressivo Maior, listados na $4^{\mathrm{a}}$ versão do DSM-IV. Esse instrumento pode ser usado em adolescentes a partir de 13 anos e em adultos. É um instrumento de autoaplicação, constituído por 21 grupos de afirmações sobre sintomas depressivos que poderiam ter ocorrido nos últimos 15 dias (GORENSTEIN et al., 2011). Cada item da escala é integrado por quatro afirmativas, com pontuação em escala Likert, variando de 0 a 3, possibilitando a soma de pontuações no intervalo entre 0 a 63 . Descreve a sintomatologia depressiva, compreendendo cognições negativas e sintomas somato-vegetativos. Os itens 16 (alterações no padrão do sono) e 18 (alterações de apetite) demandam atenção especial, pois cada um contém sete opções com valores $0,1 \mathrm{a}, 1 \mathrm{~b}, 2 \mathrm{a}, 2 \mathrm{~b}$, $3 \mathrm{a}$ e $3 \mathrm{~b}$ que são usados para diferenciar o aumento e a diminuição do comportamento ou da motivação. Em geral, a aplicação do BDI-II leva em torno de 10 a 15 minutos para ser 
respondido e sua pontuação final é classificada em níveis mínimo, leve, moderado e grave, indicando assim a intensidade da depressão (GORENSTEIN et al., 2011).

\section{Inventário de Habilidades Sociais para Adolescentes - Del Prette (IHSA-Del}

Prette): destinado a caracterizar o desempenho social de adolescentes brasileiros entre 12 e 17 anos nos vários contextos e tipos de interlocutores. Trata-se de um instrumento de autorrelato que permite avaliar o repertório de habilidades sociais em um conjunto de situações interpessoais cotidianas, considerando os indicadores de frequências e dificuldade com que os adolescentes reagem às diferentes demandas de interação social. $\mathrm{O}$ IHSA-Del Prette é composto por 38 itens que contemplam habilidades de relacionamento com diferentes interlocutores (parceiro/a afetivo-sexual, pais e irmãos, colegas, amigos, pessoas de autoridade, desconhecidos ou não especificados) que são requeridas em contexto público (escola, trabalho, lazer, consumo), privado (familiar e íntimo) ou não especificado. Para cada item, o adolescente deve se autoavaliar, julgando a dificuldade que ele apresenta na reação indicada no item e a frequência com que é apresentada (DEL PRETTE; DEL PRETTE, 2009). Nesses indicadores de frequências e dificuldades, as respostas são mensuradas em uma escala tipo Likert de 5 pontos. Quanto à frequência, as categorias de respostas são: 0 a 2 - para cada 10 situações desse tipo, comporto-me dessa forma no máximo 2 vezes; 2 a 4 - para cada 10 situações desse tipo, comporto-me dessa forma de 3 a 4 vezes; 5 a 6 - para cada 10 situações desse tipo, comporto-me dessa forma de 5 a 6 vezes; 7 a 8 - para cada 10 situações desse tipo, comporto-me dessa forma de 7 a 8 vezes; 9 a 10 - para cada 10 situações desse tipo, comporto-me dessa forma de 9 a 10 vezes. Nas categorias de respostas para indicar a dificuldade é apresentada as seguintes opções: nenhuma, pouca, média, muita e total (DEL PRETTE; DEL PRETTE, 2009).

\section{Procedimentos para coleta dos dados}

Foram realizados contatos com a direção da escola e apresentada a proposta da pesquisa, que foi acolhida pela escola, incluído professores e coordenadores. No primeiro contato com os adolescentes, foi feito o convite para participação na pesquisa. Os adolescentes que aderiram ao estudo levaram para os pais ou responsáveis a carta-convite, o 
Termo de Consentimento Livre e Esclarecido do responsável- TCLE e o Termo de Consentimento Livre e Esclarecido do adolescente-TCLE.

$\mathrm{Na}$ coleta dos dados, foram desenvolvidos os seguintes procedimentos: os estudantes participantes da turma, aproximadamente 15 alunos por sessão, foram convidados a ir à sala reservada para aplicação dos instrumentos. Nesse espaço, eles foram acomodados e, em seguida, realizou-se a aplicação dos instrumentos. A aplicação de cada instrumento foi coletiva e ocorreu sob a orientação da pesquisadora. Foram realizados os seguintes procedimentos para a coleta dos dados: distribuição e leitura das instruções dos instrumentos conforme os procedimentos padronizados, com prévios esclarecimentos das dúvidas colocadas pelos adolescentes, recolham e checagem do preenchimento dos instrumentos. Os instrumentos foram aplicados na seguinte ordem: primeiro o BDI-II, seguido do IHSA-Del Prette e, por último, a aplicação do questionário sociodemográfico.

\section{Procedimentos para análise dos dados}

Os dados do IHSA-Del Prette foram analisados por meio dos resultados brutos (escores totais e de cada escala), convertidos em posição percentílica. Esses percentis foram relacionados aos dados da amostra normativa do mesmo sexo e da mesma idade conforme as orientações especificadas no manual do instrumento (DEL PRETTE; DEL PRETTE, 2009).

As informações foram interpretadas considerando a posição percentílica do escore total do respondente, conforme a frequência em: repertório altamente elaborado de habilidades sociais (percentil entre 76-100); repertório elaborado de habilidades sociais (percentil entre 66-75); bom repertório de habilidades sociais (percentil entre 36-65); repertório médio inferior de habilidades sociais (percentil entre 26-35); e repertório abaixo da média inferior de habilidades sociais (percentil entre 01-25). Também foram analisados quanto à dificuldade na emissão das habilidades sociais, conforme as seguintes categorias: alto custo de resposta ou ansiedade na emissão das habilidades (percentil entre 66-100); médio custo de resposta ou ansiedade na emissão das habilidades (percentil entre 36-65); e baixo custo de resposta ou ansiedade na emissão das habilidades (percentil entre 01-35) (DEL PRETTE; DEL PRETTE, 2009). 
Os níveis de depressão dos participantes foram aferidos por meio da soma dos pontos de cada item do BDI-II. Conforme as orientações especificadas no manual, os participantes foram caracterizados considerando os seguintes níveis de depressão: mínimo (pontuação total 0-13); leve (pontuação total 14-19); moderado (pontuação total 20-28); e grave (pontuação total 29-63) (GORENSTEIN et al., 2011).

Foi usado o teste estatístico Coeficiente de Correlação de Pearson, na análise de correlação das variáveis sintomas depressivos e habilidades sociais de adolescentes e para verificar a direção do relacionamento, e o Teste $t$, para verificar as diferenças entre os escores de depressão, frequência e dificuldade de habilidades sociais dos adolescentes conforme o sexo. Para esses testes, adotou-se como parâmetro o nível de significância inferior a 0,05.

\section{Resultados e Discussão}

Em relação as habilidades sociais dos 153 participantes desse estudo; 19 (12,4\%) relataram repertório altamente elaborado de habilidades sociais; 50 (32,7\%) demonstraram ter repertório elaborado de habilidades sociais; 76 (49,7\%) adolescentes relataram ter bom repertório de habilidades sociais; 8 (5,2\%) apresentaram repertório abaixo da média inferior de habilidades sociais; e não houve respondentes que apresentaram repertório médio inferior de habilidades sociais. Na descrição dos sintomas depressivos dos 153 participantes desse estudo 117 (75,5\%), descreveram nível mínimo de sintomas depressivos; 27 (17,4\%) apresentaram índice leve de sintomatologia depressiva; 4 (2,6\%) relataram sintomas depressivos moderados; e 5 (3,2\%) indicaram gravidade na sintomatologia depressiva.

Foi realizada a análise de correlação de Pearson com os dados dos escores brutos de sintomas depressivos, frequência e dificuldade de habilidades sociais e das subescalas de habilidades sociais dos participantes. Encontrou-se uma correlação inversa entre sintomas depressivos e frequência de habilidades sociais $(r=-0,67 ; p<0,01)$ e uma correlação positiva entre sintomas depressivos e dificuldade de habilidades sociais $(r=0,68 ; p<0,01)$. Segundo Dancey e Reidy (2006), correlações que se aproximam de 0,70 são consideradas fortes.

A correlação negativa sugere que adolescentes com índices elevados de sintomas depressivos, tendem a suprimir comportamentos de frequência de habilidades sociais. $\mathrm{Na}$ 
correlação positiva encontrada nas variáveis sintomatologias depressiva e dificuldade na emissão de resposta ou ansiedade de resposta de habilidades sociais, os respondentes com níveis elevados de sintomas depressivos, possivelmente, demonstram problemas em contextos e situações que demandas interações sociais.

Explorando os dados de frequência e de dificuldade de habilidades sociais das subescalas, encontraram-se mais algumas correlações. Verificou-se uma correlação negativa entre sintomas depressivos e as subescalas de civilidade $(r=-0,63 ; p<0,01)$; assertividade $(r=$ - 0,62; $p<0,01)$; e abordagem afetiva $(r=-0,59 ; p<0,01)$ de frequência de habilidades sociais. Também, encontrou-se uma correlação positiva entre os dados de sintomas depressivos e os de dificuldade de habilidades sociais das subescalas de empatia $(\mathrm{r}=0,65 ; \mathrm{p}<0,01)$; civilidade $(\mathrm{r}=0,67 ; \mathrm{p}<0,01) ;$ assertividade $(\mathrm{r}=0,63 ; \mathrm{p}<0,01)$; abordagem afetiva $(\mathrm{r}=0,64 ; \mathrm{p}<0,01)$; e desenvoltura social $(\mathrm{r}=0,59 ; \mathrm{p}<0,01)$.

As correlações negativas entre sintomas depressivos e subclasses de habilidades sociais possibilitam sugerir que, adolescentes com níveis elevados de sintomas depressivos podem apresentar baixa frequência de comportamentos de civilidade, assertividade e abordagem afetiva. Além disso, as correlações positivas entre sintomatologia depressiva e dificuldade na emissão de resposta de habilidades sociais permitem aludir que, adolescentes com níveis elevados de sintomas depressivos apresentam dificuldade de interação em diferentes situações, com diferentes interlocutores, a exemplo, de professores, colegas, amigos, pais, diretores, etc. e em diferentes contextos. Porém, tais resultados devem ser considerados com cautela, uma vez que as subescalas apresentaram baixo nível de consistência interna.

As subclasses de habilidades sociais empatia, civilidade, assertividade abordagem afetiva e desenvoltura social envolvem diversos comportamentos de demanda do interlocutor ou contexto e do próprio adolescente na situação de interação social. Entre os comportamentos estão: o despedir-se de pessoas; agradecer elogios ou favores; elogiar; cumprimentar e realizar favores; defender seus direitos sem desrespeitar o interlocutor; negociar soluções em situações conflituosas; demonstrar desagrado; resistir à pressão do grupo; conversar com pessoas de autoridades; estabelecer contatos e conversações de amizade e de trabalho; expressar satisfação ou insatisfação a diferentes maneiras de carinho e relações 
de intimidade sexual; identificar sentimentos e problemas do outro; guardar segredos e preocupar se com o bem estar do outro (DEL PRETTE; DEL PRETTE, 2009).

Além disso, Del Prette e Del Prette (2010) salientaram que o funcionamento cognitivo das pessoas com depressão tem um papel importante em suas dificuldades interpessoais. E que o excesso de autocriticismo, a negação da autoeficácia, a baixa autoestima e as crenças distorcidas, geralmente estão relacionadas às dificuldades da pessoa com depressão para manter conversações interessantes, agir nas defesas dos próprios direitos e demonstrar empatia.

A análise dos dados sobre a correlação entre sintomas depressivos e as subclasses de frequência e de dificuldade de habilidades relatados pelos adolescentes corrobora a Caballo (2010) que considera os programas de Treino de Habilidades Sociais como uma estratégia eficaz para o tratamento da depressão. Assim, ele considera a premissa de que o comportamento depressivo está relacionado com o funcionamento interpessoal inadequado.

Conforme o pesquisador, esses programas centraram nos repertórios comportamentais de asserção negativa (defesa dos seus direitos e ação com base nos seus próprios interesses), asserção positiva (expressão de sentimentos positivos acerca de outras pessoas e apresentação de desculpas apropriadas) e nas habilidades de conversação (fazer perguntas, fazer autorrevelações apropriadas e encerrar conversas) que parecem ser especificamente relevantes para a pessoa com depressão.

Além das habilidades concretas, como apontam Friedberg e McClure (2004), adolescentes deprimidos podem necessitar de ser educados em comportamentos não verbais e podem se beneficiar com a leitura da linguagem corporal, com o contato visual, as observações de sinais sociais de outras pessoas.

Para fins de melhor compreensão da relação entre depressão e habilidades sociais, procedeu-se à análise dos resultados na distribuição entre as categorias de níveis de sintomas depressivos e de habilidades sociais, conforme as categorias propostas pelos autores dos instrumentos BDI-II e IHSA- Del Prette (2009). Os participantes com índices de sintomas depressivos mínimos e leves foram categorizados sem sintomas depressivos e os que apresentaram níveis moderados e graves de depressão foram considerados com sintomas depressivos. Esses resultados foram cruzados com a frequência de habilidades sociais dos participantes, categorizados em repertório elaborado, bom e inferior a médio (Tabela 1). 
Conforme esses dados, os 144 adolescentes sem sintomas depressivos, em relação à frequência de habilidades sociais, desses 69 (47,9\%) relataram elaborado repertório e 75 $(52,1 \%)$ bom repertório de habilidades sociais. Dos $9(100 \%)$ adolescentes com sintomas depressivos, $7(77,8 \%)$ relataram repertório de frequência de habilidades sociais inferior a médio e $2(22,2 \%)$ apresentaram bom repertório de frequência de habilidades sociais (Tabela $1)$.

Tabela 1 - Relação entre sintomas depressivos e frequência de habilidades sociais

\begin{tabular}{lll}
\hline $\begin{array}{l}\text { Repertório de } \\
\text { habilidades sociais }\end{array}$ & $\begin{array}{l}\text { Sem sintomas depressivos } \\
\mathbf{n} \mathbf{0}(\%)\end{array}$ & $\begin{array}{l}\text { Com sintomas depressivos } \\
\mathbf{n}(\mathbf{\%})\end{array}$ \\
\hline Elaborado & $69(47,9 \%)$ & - \\
Bom & $75(52,1 \%)$ & $2(22,2 \%)$ \\
Inferior à média & - & $7(77,8 \%)$ \\
\hline Total & $\mathbf{1 4 4 ( 1 0 0 \% )}$ & $\mathbf{9 ( 1 0 0 \% )}$ \\
\hline
\end{tabular}

A esses resultados de déficit nas interações sociais dos participantes dessa pesquisa com sintomas depressivos corroboram com Rockhill et al. (2009), ao sugerirem que adolescentes com sintomatologia depressiva demonstram baixa frequência de habilidades sociais, assim como Gresham, Cook e Crews (2004), ao apontarem que adolescentes com níveis elevados de depressão têm restritas interações sociais. E Witvliet et al. (2010) que consideraram o afastamento do adolescente dos pares, um fator crítico para o aumento da sintomatologia depressiva.

Os dados dos participantes com sintomas depressivos e sem sintomas depressivos foram cruzados com os resultados de dificuldade de resposta ou ansiedade na emissão de habilidades sociais. Verificou-se que do total de 144 adolescentes sem sintomas depressivos, 77 (55,0\%) deles têm médio custo de resposta ou ansiedade na emissão de comportamentos de habilidades sociais. E com baixo custo de resposta ou ansiedade na emissão de habilidades sociais, encontrou-se 67(45,0\%) adolescentes (Tabela 2).

Tabela 2 - Relação entre sintomas depressivos e dificuldades de habilidades sociais

\begin{tabular}{lll}
\hline $\begin{array}{l}\text { Dificuldade de } \\
\text { habilidades sociais }\end{array}$ & $\begin{array}{l}\text { Sem sintomas depressivos } \\
\mathbf{n} \mathbf{0}(\%)\end{array}$ & $\begin{array}{l}\text { Com sintomas depressivos } \\
\mathbf{n} \mathbf{( \% )}\end{array}$ \\
\hline Alto custo & - & $8(88,9 \%)$ \\
Médio custo & $77(55,0 \%)$ & $1(11,1 \%)$ \\
Baixo custo & $67(45,0 \%)$ & - \\
\hline Total & $\mathbf{1 4 4}(\mathbf{1 0 0} \%)$ & $\mathbf{9 ( 1 0 0 \% )}$ \\
\hline
\end{tabular}


Dos nove respondentes com sintomas depressivos, $8(88,9 \%)$ deles relataram alto custo de resposta ou ansiedade na emissão de habilidades sociais e, apenas, $1(11,1 \%)$ demonstrou médio custo de resposta ou ansiedade na emissão de comportamentos de habilidades sociais. Os dados apontaram que 144 adolescentes relataram médio custo de resposta ou ansiedade na emissão de habilidades sociais, aspecto que possibilita sugerir medidas psicoeducativas, com vista nas suas interações sociais enquanto elementos protetores da saúde mental e favorecedores do seu bem-estar.

$\mathrm{Na}$ adolescência, um número considerável de transformações, num período curto de tempo, possibilita certa inquietação relacionada ao desenvolvimento de problemas ligados à saúde mental (BAPTISTA; BAPTISTA; DIAS, 2001). Segrin (2000) considerou três hipóteses na relação entre habilidades sociais e depressão: o déficit de habilidades sociais como causa de depressão; a depressão como causa do déficit de habilidades sociais; e restritas habilidades sociais como fator vulnerável à depressão.

Considerando a suposição de Segrin (2000) da depressão como causa do deficitário repertório de habilidades sociais, é possível sugerir que os $2(22,2 \%)$ adolescentes dessa pesquisa com sintomas depressivos e com bom repertório de frequência de habilidades sociais, futuramente, poderão apresentar frequência inferior de habilidades sociais.

A partir do pressuposto de Segrin (2000), do deficitário repertório de habilidades sociais como possível fator de vulnerabilidade para o desenvolvimento da depressão, é possível sugerir que os $75(52,1 \%)$ adolescentes deste estudo sem depressão e com repertório moderado de frequência de habilidades sociais, encontram-se numa zona fragilizada, favorável ao desenvolvimento de sintomas depressivos a nível moderado ou grave.

$\mathrm{Na}$ análise da correlação de Pearson, os valores elevados na frequência de habilidades sociais implicaram valores baixos de sintomatologia depressiva e vice-versa. Os níveis altos de sintomas depressivos sugeriram dificuldade de emissão de resposta ou ansiedade de habilidades sociais. Os achados da presente pesquisa sugerem que há uma relação entre sintomas depressivos e repertório de habilidades sociais em adolescentes, corroborando com os achados dos estudos anteriores (CAMPOS, 2009; DEL PRETTE; DEL PRETTE, 2010; FERNANDES; FALCONE， 2009; GOLDSTEIN; MIKLOWITZ; MULLEN, 2006; 
GRESHAM; COOK; CREWS, 2004; HOROWITZ, 2007; ROCKHILL et al. 2009; SELIGMAN, 1977; TAVARES, 2005; YOUNG; MUFSON; GALLOP, 2010).

Independentemente do sexo, adolescentes com níveis elevados de sintomas depressivos poderão ter sua qualidade de vida e bem-estar comprometidos. Supõe-se que sintomas depressivos relacionados a déficits nas habilidades sociais podem prejudicar os adolescentes em áreas importantes do seu desenvolvimento, entre elas, nas relações com os pares, com os familiares, com os colegas do contexto profissional e do contexto escolar.

$\mathrm{Na}$ análise de Silva e Murta (2009), déficits em habilidades sociais na adolescência podem afetar fases posteriores do ciclo vital. Considera-se que programas com foco nas interações sociais favorecem mudanças no quadro clínico de depressão (CAMPOS, 2009; GUNLICKS-STOESSEL et al. 2010; HOROWITZ, 2007; YOUNG; MUFSON; GALLOP, 2010). Com isso sugerem-se investimentos voltados para o Treino de Habilidades Sociais dos nove adolescentes desse estudo que relataram níveis críticos de sintomas depressivos e descreveram fragilizadas interações sociais, tal intervenção poderá contribuir com a regulação emocional deles.

Além disso, é relevante pensar e propor medidas interventivas de caráter interdisciplinar, articulando as áreas de políticas públicas de saúde do adolescente, políticas educativas e projetos sociais. Destaca-se, a importância de um olhar integral para os adolescentes que demandam melhoras nas interações sociais, principalmente, as relacionadas a sintomas e indicadores de sintomas depressivos.

\section{Considerações Finais}

A presente pesquisa se propôs a descrever a relação entre sintomas depressivos e o repertório de habilidades sociais em adolescentes, relacionando a sintomatologia depressiva à frequência e dificuldades de habilidades sociais; identificar os níveis de sintomas depressivos apresentados pelos participantes; caracterizar o repertório de habilidades sociais dos adolescentes; e contribuir com a construção de conhecimento sobre a depressão na adolescência na perspectiva das interações sociais. 
Entre os achados, encontrados em conformidade com esses objetivos, aponta-se a existência de uma correlação inversa entre sintomas depressivos e frequência de habilidades sociais e uma correlação positiva entre sintomatologia depressiva e dificuldade de habilidades sociais. Assim, é possível sugerir que há relação entre sintomas depressivos e repertório de habilidades sociais de adolescentes.

Os resultados da pesquisa apresentam algumas contribuições, entre elas, possibilita sugerir o Treino de Habilidades Sociais educativas como um elemento favorecer do desenvolvimento socioemocional dos adolescentes com níveis elevados de sintomas depressivos e com fragilizadas interações sociais. Essa medida interventiva poderá amenizar conflitos, indisciplina e violência nesse contexto, uma vez que o repertório de habilidades sociais adequado favorece a competência social, respostas adaptativas em situações adversas e contribui com a elevada autoestima, o autoconceito positivo e a aprendizagem.

No Brasil, ainda há poucos estudos que focalizam essa temática, portanto, essa pesquisa contribui com o debate inicial. Contudo, não supre a lacuna de conhecimento a respeito da relação entre sintomatologia depressiva e habilidades sociais de adolescentes do contexto brasileiro.

\section{Referências}

BAPTISTA, M. N.; BAPTISTA, A. S. D.; DIAS, R. R.. Estrutura e suporte familiar como fatores de risco na depressão de adolescentes. Psicologia, Ciência, Profissão, Brasília, v. 21, n. 2, jun. 2001 Disponível em: <http://www.scielo.br/scielo.php?script=sci_arttext\&pid=S141498932001000200007\&lng=en\&nrm=iso>. Acesso em: 15 set. 2013.

CABALLO, V. E. . Manual de avaliação e treinamento das habilidades sociais. $3^{\mathrm{a}}$ reimp. São Paulo: Santos, 2010.

CAMPOS, J. R.. Habilidades sociais de adolescentes com indicadores de depressão: considerando fatores de gênero e socioeconômicos. São Carlos. 2010.101 f. Dissertação (Mestrado em Educação Especial)- Universidade Federal de São Carlos, São Carlos, 2010. Disponível em: <http://www.ppgpsi.ufscar.br/corpo-discente/todas/dissertacoes/dissjosianerosa-campos>. Acesso em: 15 out. 2012. 
. Relações entre habilidades sociais e depressão: um panorama geral.In: II Seminário Internacional de Habilidades Sociais, 2009, Rio de Janeiro. Anais do II Seminário Internacional de Habilidades sociais. Rio de Janeiro: Universidade do Estado do Rio de Janeiro, 2009. Mesa redonda. Disponível em: <http://www.bvspsi.org.br/local/file/congressos/ANAIS_II-SIHS2009.pdf>. Acesso em: 10 mar. 2013.

DANCEY, C.; REIDY, J.. Estatística sem matemática para psicologia: usando SPSS para windows. Porto Alegre, Artmed, 2006.

DEL PRETTE, A.; DEL PRETTE, Z. A. P.. Inventário de habilidades sociais para adolescentes (IHSA- Del Prette): manual de aplicação, apuração e aplicação. São Paulo: Casa do Psicólogo, 2009.

DEL PRETTE, Z. A. P.; DEL PRETTE, A.. Avaliação multidimodal de habilidades sociais em crianças: procedimentos, instrumentos e indicadores.In: BANDEIRA, M.; DEL PRETTE, Z. A. P.; DEL PRETTE, A. (Org.). Estudos sobre habilidades sociais e relacionamento interpessoal. São Paulo: Casa do Psicólogo, 2006, p. 47-68.

. Um sistema de categorias de habilidades sociais educativas. Paidéia, Ribeirão Preto, v. 18, n. 41, dez. 2008. Disponível em: <http://www.scielo.br/scielo.php? script=sci_arttext\&pid=S0103-863X2008000300008\&lng=en\&nrm=iso $>$.

Acesso em: 20 ago. 2012.

.Psicologia das habilidades sociais: terapia, educação e trabalho. 7. ed. Petrópolis: Vozes, 2010 2005

.Psicologia das habilidades sociais na infância: teoria e prática. Petrópolis: vozes,

. Treinamento de habilidades sociais: panorama geral da área. In: HAASE, V. G. et al. (Org). Psicologia do desenvolvimento: contribuições interdisciplinares. Belo Horizonte: Health, 2000.

Habilidades Sociais e educação: pesquisa e atuação em psicologia escolar/educacional. DEL PRETTE, Z. A. P. (Org.). In: Psicologia escolar e educacional, saúde e qualidade de vida. Campinas: Editora Alíneia, 3ª edição, 2008.

Habilidades Sociais: uma área em desenvolvimento. Psicologia: Reflexão e Crítica, Porto Alegre, v.9, n.2, p.287-289, 1996.

FERNANDES, C. S.; FALCONE, E. M. O.. Aspectos conceituais e empíricos das relações entre habilidades sociais, depressão e estilos de vinculação. 2009, Rio de Janeiro. In: II SEMINÁRIO INTERNACIONAL DE HABILIDADES SOCIAIS, 2009, Rio de Janeiro. Anais do II Seminário Internacional de Habilidades sociais. Rio de Janeiro: Universidade do Estado do Rio de Janeiro, 2009. Mesa redonda. Disponível em: www. <http://www.bvspsi.org.br/local/file/congressos/ANAIS_II-SIHS2009.pdf> Acesso em: 10 mar. 2013. 
FRIESERBERG, R. D.; MCCLURE J. M. A prática clínica de terapia cognitiva com crianças e adolescentes. Tradução Cristina Monteiro. Porto Alegre: Artmed, 2004.

GOLDSTEIN, T.R.; MIKLOWITZ, D.J.; MULLEN, K. . Social skills knowledge and performance among adolescents with bipolar disorder. Bipolar Disorders, n. 8, p. 350-361, 2006.

GORENSTEIN, C. et al.. Manual do Inventário de Depressão de Beck: BDI- II. Adaptação, BECK, A. T.; STEER, R. A., BROWN, G. K.. São Paulo: Casa do Psicólogo, 2011.

GRESHAM, F.M.; COOK, C.R.; CREWS, S.D. . Social skills training for children and youth with emotional and behavioral disorders. Behavioral Disorders, v. 30, n. 1, p. 32-46, Nov. 2004.

GUNLICKS-STOESSEL, M. et al. . The impact of perceived interpersonal functioning on treatment for adolescent depression: IPT-A versus treatment as usual in school-based health clinics. Journal of Consulting and Clinical Psychology, Washington, v. 78, n. 2, p. 260 267, Apr. 2010.

HOROWITZ et al. Prevention of depressive symptoms in adolescents: arandomized trial of cognitive-behavioral and interpersonal prevention programs. Journal of Consulting and Clinical Psychology, Washington, v. 75, n. 5, p. 693-706, 2007.

ROCKHILL et al.. Social competence and social support as mediators between comorbid depressive and conduct problems and functional outcomes in middle school children. Journal of Adolescence, v. 32, n. 3, p. 535-553, 2009.

SEGRIN, C.. Social skills deficits associated with depression. Clinical Psychology Review, v. 20, n. 3, pp. 379-403, 2000.

SELIGMAN, M.E.P. Desamparo: sobre depressão, desenvolvimento e morte. São Paulo: HUCITC-EDUSP. 1977.

SILVA, M.P.; MURTA, S. G.. Treinamento de habilidades sociais para adolescentes: uma experiência no programa de atenção integral à família (PAIF). Psicologia: Reflexão e Crítica, Porto Alegre, v.22, n. 1, p. 136-143, 2009. Disponível em: <http://www.scielo.br/scielo.php?script=sci_arttext\&pid=S0102$79722009000100018>$. Acesso em: 26 jun. 2013.

TAVARES, L.. Abordagem cognitivo-comportamental no atendimento de pacientes com história de depressão e déficit em habilidades sociais. São Carlos, 2005, 236 f. Relatório (Graduação em Psicologia) - Universidade Federal de São Carlos, São Carlos, 2005. Disponível em: 〈http://newpsi.bvs-psi.org.br/tcc/83.pdf〉. Acesso em: 10 jun. 2012. 
WITVLIET et al.. Early adolescent depressive symptoms: prediction from clique isolation, loneliness, and perceived social acceptance. Journal of Abnormal Child Psychology, n. 38, p. 1045-1056, May, 2010.

YOUNG, J. F.; MUFSON, L.; GALLOP, R. Preventing depression: a randomized trial of interpersonal psychotherapy-adolescent skills training. Depression and anxiety, New York, v. 27, n.5, p.426-433, May, 2010.

\section{Como citar este artigo (Formato ABNT):}

SANTANA, Maria Luzia S.; FUKUDA, Cláudia C.; CARVALHO, Erenice N.S. A Relação entre Sintomas Depressivos e Habilidades Sociais em Adolescentes. Id on Line Revista Multidisciplinar e de Psicologia, Julho de 2017, vol.11, n.36, p.295-312. ISSN: 1981-1179.

Recebido: 11.07.2017

Aceito: 14.07.2017 